\title{
Adaptación española de una intervención online para el tratamiento del insomnio: el programa SHUTI
}

\author{
ÁNGEL ENRIQUE \\ aenrique@uji.es \\ SANDRA FERnÁNDEZ REBOLL \\ al340121@uji,es \\ LEE RITTERBAND \\ LR5B@hscmail.mcc.virginia.edu \\ CRISTINA BOTELLA \\ botella@uji.es
}

\section{Resumen}

Introducción: El insomnio es un problema de gran envergadura que afecta a millones de personas en todo el mundo. En España, se ha comprobado que el 6,4 \% de la población cumpliría criterios para su diagnóstico. Los estudios sobre el tema han demostrado que la terapia cognitivo-conductual para insomnio (TCC-I) es muy efectiva; sin embargo, no todas personas tienen acceso a dicho tratamiento. En este sentido, el tratamiento a través de Internet facilitaría la diseminación. El programa SHUTi (Sleep Healthy Using the Internet) es un programa online personalizado para el tratamiento del insomnio compuesto por seis módulos que abordan las estrategias fundamentales de la TCC-I: psicoeducación, restricción de sueño, control estimular, higiene del sueño, reestructuración cognitiva y prevención de recaídas. SHUTi ya ha demostrado su eficacia en el tratamiento del insomnio a través de Internet en diferentes países anglosajones. Objetivo: El objetivo del presente trabajo es describir este programa online, presentar la estrategia seguida para su adaptación y traducción y, finalmente, analizar las implicaciones futuras del mismo. Método: El proceso de adaptación se ha realizado manteniendo la misma estructura de tratamiento y una traducción ajustada a la lengua española, que ha sido revisada por expertos y en estrecho contacto con los autores del programa. Discusión: La adaptación se ha realizado con éxito y el programa está a punto para ser aplicado. Dado que es un estudio en progreso, todavía no se dispone de resultados de la aplicación del programa en muestra española, pero se espera poder replicar los resultados obtenidos en estudios previos.

Palabras clave: insomnio, internet, tratamiento cognitivo conductual, traducción y adaptación al español, intervención online. 


\section{Abstract}

Introduction: Insomnia is a major problem that affects millions of people worldwide. In Spain, $6.4 \%$ of the population would meet the criteria to be diagnosed it. Studies have shown that Cognitive-Behavioural Therapy (СВT) for insomnia is very effective, but not everyone has access to this treatment. Hence treatment over the Internet would facilitate its dissemination. SHUTi (Sleep Healthy Using the Internet) is an online tailored programme for insomnia treatment that comprises six modules that encompass essential CBT-I strategies: psycho-education, sleep restriction, stimulus control, sleep hygiene, cognitive restructuring and relapse prevention. SHUTi has already shown its efficacy in insomnia treatment over the Internet in different Englishspeaking countries. The present work aims to describe the online programme, explain the strategy followed to adapt and translation it, and discuss its future implications. Method: The adaptation process was done by maintaining the same treatment structure and an adjusted translation to Spanish. It was reviewed by experts in close connection with the programme's authors. Discussion: Adaptation proved an absolute success and the programme is ready to be implemented. As work is underway, the results are not yet available in the Spanish sample, but those obtained in previous studies are expected to be replicated.

Keywords: Insomnia, Internet, Cognitive behavioural treatment, translation and adaptation into Spanish, online intervention

\section{Introducción}

El insomnio es un problema de gran envergadura que afecta a millones de personas en todo el mundo. En España, se ha comprobado que el 6,4 \% de la población cumpliría criterios para su diagnóstico y que 1 de cada 5 tienen problemas de sueño al menos 3 noches por semana (Ohayon y Sagales, 2010), siendo esta prevalencia mayor en mujeres (23,9 \%) que en hombres (17,6\%). Además, también se ha comprobado que los problemas de sueño aumentan con la edad, llegando a prevalencias de entorno a un $40 \%$ en personas mayores de 65 años (Ohayon y Sagales, 2010). A pesar de las altas tasas de prevalencia, los datos indican que de las personas con problemas de insomnio, tan solo entre un $15 \%$ y un $25 \%$ demandan ayuda por esta problemática (Mellinger, Balter y Unlenhuth, 1985; Ohayon y Sagales, 2010). En este sentido, la asistencia recibida ante esta patología suele ser pautar psicofármacos para dormir (Morin, LeBlanc, Daley, Gregoire y Merette, 2006). Aunque la medicación para el sueño ha demostrado ser efectiva a corto plazo para el tratamiento del insomnio agudo, su uso está asociado con diferentes limitaciones, tales como riesgo de dependencia, una serie de efectos secundarios y una disminución del efecto terapéutico con el tiempo, así como alteraciones en los estadios de sueño (Holbrook, Crowther, Lotter, Cheng y King, 2000).

La terapia cognitivo-conductual para insomnio (TCC-I) es una alternativa de tratamiento no farmacológico dirigida a la restauración de condutas desadaptativas y pensamientos disfuncionales que funcionan como mantenedores del insomnio. La TCC-I ha demostrado ser uno de los tratamientos más efectivos frente al insomnio (Morin y cols., 2006). De hecho, un reciente metanálisis sobre la eficacia de la TCC-I en adultos con insomnio crónico ha indicado que 
se producían mejoras clínicamente significativas en los diarios de sueño de estas personas (Trauer, Qian, Doyle, Rajaratnam y Cunnington, 2015). Además, las mejoras provocadas por la TCC-I han sido asociadas a beneficios más duraderos y mayor satisfacción con los pacientes, siendo valorada como más efectiva y aceptable que la medicación (Morin, Gaulier, Barry y Kowatch, 1992).

A pesar de los buenos resultados generados por la TCC-I, no todas las personas tienen acceso al tratamiento ya sea por dificultad en encontrar clínicos competentes o por dificultades geográficas (Edinger y Means, 2005). En este sentido, el tratamiento a través de Internet podría ser un modo efectivo y eficiente para superar estas barreras, permitiendo una mayor diseminación y alcance del mismo. Frente a esta necesidad, se han elaborado diferentes programas dirigidos al tratamiento del insomnio a través de Internet, los cuales han demostrado eficacia y se han posicionado como una alternativa viable para el tratamiento del insomnio (Zachariae, Lyby, Ritterband y O'Toole, 2016). No obstante, ninguno de ellos se ha elaborado o adaptado a la lengua española, por lo que actualmente en España no se cuenta con tratamientos autoaplicados para el insomnio a través de Internet. El programa SHUTi (Sleep Healthy Using the Internet) es un programa online personalizado para el tratamiento del insomnio basado en la TCC-I, que ha demostrado su eficacia en el tratamiento del insomnio a través de Internet en diferentes países anglosajones (Christensen y cols., 2016). El objetivo es, por una parte, describir detalladamente este programa y la metodología seguida para su traducción y, por otra, analizar las posibles implicaciones futuras que puede tener la aplicación del programa en población española.

\section{Método}

\section{Descripción del sistema}

El programa SHUTi ha sido diseñado poniendo mucho énfasis en los aspectos de usabilidad y apariencia del programa, con la finalidad de crear un entorno online que sea interesante, efectivo y seguro. Consta de tres partes fundamentales: la página de inicio, el diario de sueño y los módulos de tratamiento.

\section{Página de inicio}

Los usuarios acceden al programa a través de una URL en la que tienen que introducir un usuario y una contraseña personal e intransferible que previamente se les habrá creado. Una vez dentro, el usuario encuentra una página de inicio personalizada que le proporciona información basada en el estado de progreso (por ejemplo, un usuario nuevo tendría una página de bienvenida e instrucciones sobre cómo empezar con el programa, mientras que un usuario avanzado encontrría información sobre completar la siguiente tarea asignada). En la ventana principal también encontrará enlaces de acceso a las secciones principales, tales como los diarios de sueño, los módulos y el centro de impresión (para imprimir todos los recursos disponibles hasta el momento). 


\section{Diarios de sueño}

El programa SHUTi se apoya en la información introducida en los diarios de sueño. Este diario consiste en 10 preguntas acerca de la duración de las siestas, hora de acostarse y de despertarse, minutos empleados para dormirse, número de despertares nocturnos, duración de los mismos y uso de medicación o ayudas para dormir. Además, también se valora el grado de cansancio al despertar y la calidad de sueño percibida. Toda esta información es usada por el sistema para calcular la eficiencia de sueño del usuario y calcular ventanas de sueño (asignación de una hora de acostarse y levantarse que es el horario indicado para dormir) personalizadas y basadas en la eficiencia de sueño de la semana anterior.

La ventana de sueño es un tipo de restricción de sueño que implica limitar la cantidad de tiempo que se pasa en la cama para que esta coincida con la cantidad real de tiempo que el paciente duerme con el fin de regular el ciclo vigilia-sueño del paciente y mejorar su eficiencia de sueño.

Al igual que en el tratamiento convencional, la ventana de sueño cambia en función de las mejoras en la eficiencia de sueño, de manera que si esta no aumenta, tampoco cambiará la ventana. Si los usuarios no introducen los diarios requeridos, no reciben nuevas ventanas de sueño, lo cual dificultará el avance en el programa. Para el cálculo de las ventanas de sueño, el programa utiliza algoritmos que determinan si la ventana ha de incrementar, disminuir o mantenerse igual. Los usuarios seleccionan la hora deseada para levantarse y el programa asigna la ventana.

\section{Módulos de tratamiento}

El contenido y la lógica de los componentes de tratamiento están basados en la TCC-I, cara a cara, para el insomnio (Morin, 1993; Morin, 1996). Consta de seis módulos en los que se trabajan los elementos esenciales de tratamiento, incluyendo técnicas conductuales, cognitivas y educacionales. Los módulos se habilitan semanalmente tras completar el módulo anterior y requieren entre 45 y 60 minutos para ser completados.

El programa implica un alto nivel de interactividad y personalización. Además de las ventanas de sueño, la personalización se observa también a nivel de objetivos planteados. Así, al prinicipio del programa se les pide a los usuarios que se planteen objetivos específicos para diferentes aspectos de su sueño, tales como el número de horas que se pretende dormir, número de despertares nocturnos y tiempo despierto durante los despertares. Estos mismos objetivos pueden ser reevaluados durante el período de tratamiento y se presentan de nuevo al final del mismo para ver si han sido conseguidos. Además, todos los módulos cuentan con viñetas interactivas en las que se plantean ejercicios al usuario para que ponga en práctica lo aprendido. Por último, cabe destacar que durante el tratamiento se incluyen cinco experiencias (unas en formato vídeo y otras en texto) de usuarios simulados que acompañarán al usuario real a lo largo del programa. En ellas se mostrarán algunas dificultades que el usuario puede ir encontrando a lo largo del programa, de manera que este se sienta identificado y vea qué hacer cuando aparecen las dificultades.

A continuación se describen brevemente los seis módulos de tratamiento.

- Preparándote: Módulo psicoeducativo en el que se aborda la definición del insomnio y sus tipos, tasas de prevalencia, factores de riesgo e impacto del insomnio. También se establecen los objetivos de tratamiento; el módulo concluye subrayando la idoneidad y la efectividad de la TCC-I. 
- Ventana de sueño: En este módulo se introduce la restricción de sueño y el control estimular, este último a través de la inclusión de algunas reglas básicas. Este módulo se introduce la misma semana que el módulo anterior e incluye, además, información acerca de los malos hábitos de sueño, el ciclo del insomnio crónico, la lógica de la restricción de sueño, la explicación de la eficiencia de sueño y de los ajustes en la ventana de sueño.

- Conductas: En este módulo se amplía el control estimular, añadiendo más reglas básicas. Entre ellas se plantea: ir a la cama solo cuando se sienta somnolencia, levantarse de la cama si no se concilia el sueño, mantener una hora regular de acostarse y levantarse, usar la cama solo para dormir y no hacer siestas.

- Pensamientos: Este módulo incluye información acerca de la influencia de los pensamientos en la falta de sueño, se introduce reestructuración cognitiva y el ajuste de expectativas del tratamiento. Fundamentalmente, este módulo está dirigido al cambio de aquellas creencias y pensamientos negativos relacionados con el sueño que pueden exacerbar las dificultades para dormir. También se revisan los principales mitos acerca del sueño y se trabaja la catastrofización.

- Higiene del sueño: En este módulo los usuarios aprenden sobre determinados factores del estilo de vida (cafeína, nicotina, dieta, alcohol, ejercicio) y ambientales (temperatura de la habitación, ruido, luz, calidad del aire, comodidad de la cama y de la ropa de cama) que pueden afectar al sueño.

- Mirando al futuro: El último módulo es de prevención de recaídas y está dirigido a identificar situaciones de riesgo, consolidar los efectos conseguidos y revisar las técnicas aprendidas. Se proporcionan las instrucciones de cómo continuar ajustando la ventana de sueño y se diferencia entre tropiezo y recaída, preparando al usuario para ambas situaciones. También se les anima a continuar usando SHUTi si es necesario.

\section{Procedimiento}

El proceso de adaptación del programa sHUTi se ha realizado manteniendo siempre la misma estructura de tratamiento y ha sido revisada por clínicos expertos y manteniendo un contacto muy estrecho con los autores del programa. De hecho, el principal responsable de la traducción del sistema llevó a cabo la adaptación y traducción del programa en el laboratorio de los autores que desarrollaron el trabajo original. Este trabajo se desarrolló en un total de seis meses.

En todo momento, el proceso de adaptación del programa ha conllevado el cambio de determinados contenidos en los que se presentaban, entre otros, productos alimenticios y marcas de medicamentos, que se han sustituido por otras marcas y alimentos propios de la cultura y el mercado español. Los cambios referentes a todos estos productos fueron consultados con el autor original, de manera que los productos fueran lo más similares posible en cuanto a características y popularidad.

En cuanto al proceso de traducción, la estructura del programa consta de diferentes apartados. En concreto, el contenido a traducir se ha repartido entre la adaptación del texto base del programa, la traducción del contenido de los visores de pantalla interactivos, a través de los cuales el usuario contesta breves preguntas o completa diferentes juegos, subtitulado de vídeos con actores representando escenas de personas con insomnio, la adaptación de cuestionarios, así como la grabación de voces en español para simular el testimonio de pacientes con insomnio. 


\section{Resultados}

Consideramos que el proceso de traducción y adaptación del programa SHUTi al español ha culminado con éxito y en estos momentos el programa está preparado para poder ser utilizado en población española.

\section{Discusión y conclusiones}

El insomnio es un problema relevante de salud clínica con pocos recursos de tratamiento no farmacológico. El programa SHUTi ha sido diseñado para adaptar la TCC-I cara a cara en una programa autoaplicado a través de Internet (Thorndike y cols., 2008). El presente estudio ha recogido de manera detallada las características y los contenidos de este programa, así como los pasos seguidos para la traducción y la adaptación de este programa al español. Puede afirmarse que este proceso ha culminado con éxito.

Tal como se ha comentado, SHUTi es un sistema interactivo que personaliza sus recomendaciones para cada usuario a través de la introducción de datos como los diarios de sueño y el listado de síntomas. Estudios previos han demostrado que SHUTi ha sido bien aceptado por los usarios, mostrando además eficacia en la mejora del insomnio y otros síntomas comórbidos (Ritterband y cols., 2009; Thorndike y cols., 2008). Además, SHUTi también ha demostrado ser eficaz en la prevención de episodios depresivos, así como en otras poblaciones como supervivientes de cáncer (Christensen y cols., 2016; Ritterband y cols., 2012).

Actualmente en España no se cuenta con ningún programa autoaplicado a través de Internet para el tratamiento del insomnio. Dada la eficacia encontrada en los estudios mencionados, la adaptación del programa a la cultura y la lengua española abre la puerta a la posibilidad de aplicación de este tratamiento en España con el fin de replicar los resultados obtenidos y explorar la posibilidad de implementación de este programa en otras poblaciones y entornos, como atención primaria o diferentes poblaciones con insomnio como síntoma comórbido.

\section{Referencias bibliográficas}

Christensen, H., Batterham, P. J., Gosling, J. A., Ritterband, L. M., Griffiths, K. M., Thorndike, F. P., Glozier, N., O'Dea, B., Hickie, I. B. y Mackinnon, A. J. (2016). Effectiveness of an online insomnia program (SHUTi) for prevention of depressive episodes (the GoodNight Study): a randomised controlled trial. The Lancet Psychiatry, 3, 333-341.

Edinger, J. D. y Means, M. K. (2005). Cognitive-behavioral therapy for primary insomnia. Clinical Psychology Review, 25, 539-558.

Holbrook, A. M., Crowther, R., Lotter, A., Cheng, C. y King, D. (2000). Meta-analysis of benzodiazepine use in the treatment of insomnia. Canadian Medical Association Journal, 162, 225-233.

Mellinger, G. D., Balter, M. B. y Uhlenhuth, E. H. (1985). Insomnia and its treatment: prevalence and correlates. Archives of general psychiatry, 42, 225-232.

Morin, C. M., LeBlanc, M., Daley, M., Gregoire, J. P. y Merette, C. (2006). Epidemiology of insomnia: prevalence, self-help treatments, consultations, and determinants of help-seeking behaviors. Sleep medicine, 7, 123-130.

Morin, C. M., Gaulier, B., Barry, T. y Kowatch, R. A. (1992). Patients' acceptance of psychological and pharmacological therapies for insomnia. Sleep: Journal of Sleep Research \& Sleep Medicine, 15, 302-305. 
Ohayon, M. M. y Sagales, T. (2010). Prevalence of insomnia and sleep characteristics in the general population of Spain. Sleep medicine, 11, 1010-1018.

Ritterband, L. M., Bailey, E. T., Thorndike, F. P., Lord, H. R., Farrell-Carnahan, L. y Baum, L. D. (2012). Initial evaluation of an Internet intervention to improve the sleep of cancer survivors with insomnia. Psycho-Oncology, 21, 695-705.

Thorndike, F. P., Saylor, D. K., Bailey, E. T., Gonder-Frederick, L., Morin, C. M. y Ritterband, L. M. (2008). Development and perceived utility and impact of an internet intervention for insomnia. E-journal of applied psychology: clinical and social issues, 4, 32.

Trauer, J. M., Qian, M. Y., Doyle, J. S., Rajaratnam, S. M. y Cunnington, D. (2015). Cognitive behavioral therapy for chronic insomnia: a systematic review and meta-analysis. Annals of internal medicine, 163, 191-204.

Zachariae, R., Lyby, M. S., Ritterband, L. M. y O'Toole, M. S. (2016). Efficacy of internet-delivered cognitive-behavioral therapy for insomnia - A systematic review and meta-analysis of randomized controlled trials. Sleep medicine reviews, 30, 1-10. 